\title{
Direct Weight Optimization for Approxi- mately Linear Functions: Optimality and Design
}

Alexander Nazin, Jacob Roll, Lennart Ljung

Division of Automatic Control

E-mail: nazine@ipu.rssi.ru, roll@isy.liu.se,

ljung@isy.liu.se

14th June 2007

Report no.: LiTH-ISY-R-2804

Accepted for publication in SYSID'06

Address:

Department of Electrical Engineering

Linköpings universitet

SE-581 83 Linköping, Sweden

WWW: http://www.control.isy.liu.se

AUTOMATIC CONTROL

REGLERTEKNIK

LINKÖPINGS UNIVERSITET

Technical reports from the Automatic Control group in Linköping are available from http://www. control.isy.liu.se/publications. 


\begin{abstract}
The Direct Weight Optimization (DWO) approach to estimating a regression function is studied here for the class of approximately linear functions, i.e., functions whose deviation from an affine function is bounded by a known constant. Upper and lower bounds for the asymptotic maximum MSE are given, some of which also hold in the non-asymptotic case and for an arbitrary fixed design. Their coincidence is then studied. Particularly, under mild conditions, it can be shown that there is always an interval in which the DWO-optimal estimator is optimal among all estimators. Experiment design issues are also studied.
\end{abstract}

Keywords: Non-parametric identification, Function approximation, Minimax techniques, Quadratic programming, Nonlinear systems, Mean-square error 


\title{
Direct Weight Optimization for Approximately Linear Functions: Optimality and Design
}

\author{
Alexander Nazin, Jacob Roll, Lennart Ljung ${ }^{\dagger}$
}

\begin{abstract}
The Direct Weight Optimization (DWO) approach to estimating a regression function is studied here for the class of approximately linear functions, i.e., functions whose deviation from an affine function is bounded by a known constant. Upper and lower bounds for the asymptotic maximum MSE are given, some of which also hold in the non-asymptotic case and for an arbitrary fixed design. Their coincidence is then studied. Particularly, under mild conditions, it can be shown that there is always an interval in which the DWO-optimal estimator is optimal among all estimators. Experiment design issues are also studied.
\end{abstract}

Keywords: Non-parametric identification, Function approximation, Minimax techniques, Quadratic programming, Nonlinear systems, Mean-square error

\section{Introduction and Problem Statement}

Non-linear black box models of dynamical systems have long been of central interest in system identification, see, e.g., the survey Sjöberg et al. (1995). In the control community mostly models of function expansion type have been applied, like Artificial Neural Network (ANN) models, wavelets, and (neuro)fuzzy models (see, e.g., Harris et al. (2002), Suykens et al. (2002)).

Direct Weight Optimization (DWO) Roll (2003); Roll et al. (2002, 2005a,b) is a non-parametric approach to nonlinear system identification, where the unknown system function is estimated pointwise by minimizing an upper bound on the mean-square error (MSE).

In what follows, we study the particular problem of estimating an unknown univariate function $f_{0}:[-0.5,0.5] \rightarrow \mathbb{R}$ at a fixed point $\varphi^{*} \in[-0.5,0.5]$ from the given dataset $\{\varphi(t), y(t)\}_{t=1}^{N}$ with

$$
y(t)=f_{0}(\varphi(t))+e(t), \quad t=1, \ldots, N
$$

where $\{e(t)\}_{t=1}^{N}$ is a random sequence of uncorrelated, zero-mean Gaussian variables with a known constant variance $E e^{2}(t)=\sigma^{2}>0$.

\footnotetext{
*Institute of Control Sciences, Profsoyuznaya str., 65, 117997 Moscow, Russia, e-mail: nazine@ipu.rssi.ru

${ }^{\dagger}$ Div. of Automatic Control, Linköping University, SE-58183 Linköping, Sweden, e-mail: roll, ljung@isy.liu.se
} 
Here, DWO for the class of approximately linear functions is studied. This class $\mathcal{F}_{1}(M)$ consists of functions whose deviation from an affine function is bounded by a known constant $M>0$ :

$$
\begin{aligned}
& \mathcal{F}_{1}(M)=\{f:[-0.5,0.5] \rightarrow \mathbb{R} \\
& \left.\quad\left|f(\varphi)=\theta_{1}+\theta_{2} \varphi+r(\varphi), \theta \in \mathbb{R}^{2},\right| r(\varphi) \mid \leq M\right\}
\end{aligned}
$$

The DWO-estimator $\widehat{f}_{N}\left(\varphi^{*}\right)$ is defined by

$$
\widehat{f}_{N}\left(\varphi^{*}\right)=\sum_{t=1}^{N} w_{t} y(t)
$$

where the weights $w=\left(w_{1}, \ldots, w_{N}\right)^{T}$ are chosen to minimize an upper bound on $U_{N}(w)$ on the maximum MSE:

$$
U_{N}(w) \geq \sup _{f_{0} \in \mathcal{F}_{1}(M)} E_{f_{0}}\left(\widehat{f}_{N}\left(\varphi^{*}\right)-f_{0}\left(\varphi^{*}\right)\right)^{2}
$$

It can be shown Roll et al. (2005b) that the RHS of (4) is infinite unless the following constraints are satisfied:

$$
\sum_{t=1}^{N} w_{t}=1, \quad \sum_{t=1}^{N} w_{t} \varphi(t)=\varphi^{*}
$$

Under these constraints, on the other hand, we can choose the following upper bound:

$$
U_{N}(w)=\sigma^{2} \sum_{t=1}^{N} w_{t}^{2}+M^{2}\left(1+\sum_{t=1}^{N}\left|w_{t}\right|\right)^{2} \rightarrow \min _{w}
$$

See Roll et al. (2005b) for further details. A solution to the convex optimization problem $(6),(5)$ is denoted by $w^{*}$, and its components $w_{t}^{*}$ are called the DWOoptimal weights. The corresponding estimate is also called DWO-optimal. Note that (3) is a non-parametric estimator, since the parameter number $N$ is in fact the number of samples (see, e.g., Juditsky et al. (1995)). A similar approach has also been proposed in Sacks and Ylvisaker (1978) for estimating a linear part $\theta^{T} F(\varphi)$ of an unknown function $f(\varphi)=\theta^{T} F(\varphi)+r(\varphi)$ from the class $\mathcal{F}_{1}(M)$, when $r(\varphi(t))$ are treated as unknown but bounded disturbances.

The main study here is devoted to an arbitrary fixed design $\{\varphi(t)\}_{t=1}^{N}$ having at least two different regressors $\varphi(t)$. We also assume that $\varphi(t) \neq \varphi^{*}, t=$ $1, \ldots, N$, for the sake of simplicity. Further details are then given for equidistant design, i.e.,

$$
\varphi(t)=-0.5+t / N, \quad t=1, \ldots, N
$$

We also discuss the extension to uniform random design when regressors $\varphi(t)$ are uniformly distributed on $[-0.5,0.5]$, i.i.d. random variables, and $\{e(t)\}_{t=1}^{N}$ being independent of $\{\varphi(t)\}_{t=1}^{N}$.

The objective of this paper is twofold. We first find an MSE minimax lower bound among arbitrary estimators (Section 2.1). Then we study both the DWOoptimal weights $w_{t}^{*}$ and the DWO-optimal MSE upper bound $U_{N}\left(w^{*}\right)$, and the upper and lower bounds are compared (Section 2.2). Experiment design issues 
are also studied (Section 3). As we will see, some of the results hold for an arbitrary fixed design $\{\varphi(t)\}$ and a fixed number of observations $N$ while others are of asymptotic consideration, as $N \rightarrow \infty$, and of equidistant (or uniform random) design. Particularly, under equidistant design the upper and lower bounds coincide when $\left|\varphi^{*}\right|<1 / 6$ which is exactly when the DWO-optimal weights are positive.

The results presented here are extensions of those from the technical report Nazin et al. (2003).

\section{DWO-estimator: Upper and Lower Bounds}

The results in this section may be immediately extended also to multivariate functions $f: D \subset \mathbb{R}^{d} \rightarrow \mathbb{R}$. However, for the sake of simplicity, we consider below the case of $d=1$.

\subsection{Minimax Lower Bound}

Consider an arbitrary estimator $\widetilde{f}_{N}=\widetilde{f}_{N}\left(y_{1}^{N}, \varphi_{1}^{N}\right)$ for $f_{0}\left(\varphi^{*}\right)$, i.e., an arbitrary measurable function of the observation vectors $y_{1}^{N}=(y(1), \ldots, y(N))^{T}$ and $\varphi_{1}^{N}=(\varphi(1), \ldots, \varphi(N))^{T}$. Introduce

$$
e_{1}=\left(\begin{array}{ll}
1 & 0
\end{array}\right)^{T}
$$

and the shifted regressors

$$
\widetilde{\varphi}(t)=\varphi(t)-\varphi^{*} .
$$

Assertion 2.1. For any $N>1$, any estimator $\widetilde{f}_{N}$, and an arbitrary fixed design the following lower bound holds true:

$$
\sup _{f_{0} \in \mathcal{F}_{1}(M)} E_{f_{0}}\left(\widetilde{f}_{N}-f_{0}\left(\varphi^{*}\right)\right)^{2} \geq 4 M^{2}+e_{1}^{T} J_{N}^{-1} e_{1} .
$$

Here the information matrix

$$
J_{N}=\frac{1}{\sigma^{2}} \sum_{t=1}^{N}\left(\begin{array}{cc}
1 & \widetilde{\varphi}(t) \\
\widetilde{\varphi}(t) & \widetilde{\varphi}^{2}(t)
\end{array}\right)
$$

is supposed to be invertible (i.e., there are at least two different $\varphi(t)$ in the dataset). Particularly, under equidistant design (7), as $N \rightarrow \infty$,

$$
\begin{aligned}
\sup _{f_{0} \in \mathcal{F}_{1}(M)} & E_{f_{0}}\left(\widetilde{f}_{N}-f_{0}\left(\varphi^{*}\right)\right)^{2} \\
& \geq 4 M^{2}+\frac{\sigma^{2}}{N}\left(1+12 \varphi^{* 2}\right)+O\left(N^{-2}\right)
\end{aligned}
$$

Proof. Notice, that for $f_{0} \in \mathcal{F}_{1}(M)$ the observation model (1) reduces to

$$
y(t)=\theta_{1}+\theta_{2} \widetilde{\varphi}(t)+\widetilde{r}(\varphi(t))+e(t)
$$

with $\theta_{1}=f_{0}\left(\varphi^{*}\right), \theta_{2} \in \mathbb{R}$, and

$$
\widetilde{r}(\varphi(t))=r(\varphi(t))-r\left(\varphi^{*}\right), \quad|\widetilde{r}(\varphi(t))| \leq 2 M
$$


In other words, the initial problem is reduced to the one of estimating a constant parameter $\theta_{1}=f_{0}\left(\varphi^{*}\right)$ from the measurements (11) corrupted by both Gaussian $e(t)$ and non-random unknown but bounded noise $\widetilde{r}(\varphi(t))$.

Let $q(\cdot)$ denote the p.d.f. of $N\left(0, \sigma^{2}\right)$. Then the probability density of $y_{1}^{N}$ is

$$
p\left(y_{1}^{N} \mid f_{0}\right)=\prod_{t=1}^{N} q\left(y(t)-\theta_{1}-\theta_{2} \widetilde{\varphi}(t)-\widetilde{r}(\varphi(t))\right)
$$

Now,

$$
\begin{aligned}
\sup _{f_{0} \in \mathcal{F}_{1}(M)} & E_{f_{0}}\left(\widetilde{f}_{N}-f_{0}\left(\varphi^{*}\right)\right)^{2} \\
& \geq \sup _{\theta} \sup _{|\widetilde{r}| \leq 2 M} E_{\theta, \widetilde{r}}\left(\widetilde{f}_{N}-\theta_{1}\right)^{2}
\end{aligned}
$$

where $\theta=\left(\theta_{1} \theta_{2}\right)^{T}$ and the last supremum in the RHS is taken over all constant functions $\widetilde{r}(\varphi) \equiv \widetilde{r},|\widetilde{r}| \leq 2 M$, and the expectation therein is taken over probability density (13) with $\theta_{1}=f_{0}\left(\varphi^{*}\right)$ and $\widetilde{r}(\varphi) \equiv \widetilde{r}$. Applying the auxiliary Lemma A.1 with $h=e_{1}$ we arrive at the inequality (8). Consequently, (10) directly follows from (8).

Remark 2.1. The result of (10) is presented in asymptotical form. However, the term $O\left(N^{-2}\right)$ in (10) can be given explicitly as a function of $N$.

Remark 2.2. If Lemma A.2 would be applied instead of Lemma A.1 in the proof of Assertion 2.1, then the same MSE minimax lower bound (10) could be obtained for the uniform random design (and $f_{0} \in \mathcal{F}_{1}(M)$ ), even nonasymptotically, for any $N>1$ with the term $O\left(N^{-2}\right) \equiv 0$ in (10).

Remark 2.3. Assertion 2.1 may be extended to non-Gaussian i.i.d. noise sequences $\{e(t)\}$ having a regular probability density function $q(\cdot)$ for $e(t)$. Then, as is seen from the proof, the noise variance $\sigma^{2}$ in (9) and (10) should be replaced by the inverse Fisher information $I^{-1}(q)$ where

$$
I(q)=\int \frac{{q^{\prime}}^{2}(u)}{q(u)} d u
$$

\subsection{DWO-Optimal Estimator}

Following the DWO approach we are to minimize the MSE upper bound (6) subject to the constraints (5). The solution to this optimization problem as well as its properties will be dependent of $\varphi^{*}$. It turns out that there arise two different cases which are studied below separately.

\subsubsection{Positive Weights}

When all the DWO-optimal weights are positive, the following assertion shows that the lower bound is then reached.

Assertion 2.2. Let $N>1$, and $\{\varphi(t)\}_{t=1}^{N}$ be a fixed design where $J_{N}$ given by (9) is invertible, i.e., there are at least two different $\varphi(t)$. Assume that all the 
DWO-optimal weights $w_{t}^{*}$ are positive. Then the DWO-optimal upper bound for the function class (2) equals

$$
U_{N}\left(w^{*}\right)=4 M^{2}+e_{1}^{T} J_{N}^{-1} e_{1}
$$

Particularly, when

$$
\left|\varphi^{*}\right|<1 / 6
$$

the equidistant design (7) reduces (16) to

$$
\begin{aligned}
& U_{N}\left(w^{*}\right) \\
& =4 M^{2}+\left(1+12 \varphi^{* 2}\right)\left(\sigma^{2} N^{-1}+O\left(N^{-2}\right)\right)
\end{aligned}
$$

as $N \rightarrow \infty$, with the DWO-optimal weights

$$
w_{t}^{*}=\frac{1+12 \varphi^{*} \varphi(t)}{N}\left(1+O\left(N^{-1}\right)\right), t=1, \ldots, N
$$

being positive for sufficiently large $N$.

Proof. When the DWO-optimal solution $w^{*}$ only contains positive components, it is easy to see from $(6),(5)$ that the following optimization problem will have the same optimal solution:

$$
\sum_{t=1}^{N} w_{t}^{2} \rightarrow \min _{w}
$$

subject to the constraints (5). Moreover, the inverse statement holds: If the solution $w^{o p t}$ to the optimization problem (20), (5) has only positive components, then $w^{*}=w^{o p t}$.

Now, to prove (16), one needs to minimize $\|w\|_{2}^{2}$ subject to the constraints (5). Applying the Lagrange function technique, we arrive at

$$
w_{t}^{*}=\lambda+\mu \widetilde{\varphi}(t), \quad t=1, \ldots, N
$$

with

$$
\begin{aligned}
\left(\begin{array}{l}
\lambda \\
\mu
\end{array}\right) & =\left(\sum_{t=1}^{N}\left(\begin{array}{cc}
1 & \widetilde{\varphi}(t) \\
\widetilde{\varphi}(t) & \widetilde{\varphi}^{2}(t)
\end{array}\right)\right)^{-1}\left(\begin{array}{l}
1 \\
0
\end{array}\right) \\
& =\frac{1}{D_{N}} \sum_{t=1}^{N}\left(\begin{array}{c}
\widetilde{\varphi}^{2}(t) \\
-\widetilde{\varphi}(t)
\end{array}\right) \\
D_{N} & =N \sum_{t=1}^{N} \widetilde{\varphi}^{2}(t)-\left(\sum_{t=1}^{N} \widetilde{\varphi}(t)\right)^{2}
\end{aligned}
$$

Thus, from (9) and (22) follows

$$
\sum_{t=1}^{N} w_{t}^{* 2}=\lambda=\frac{1}{D_{N}} \sum_{t=1}^{N} \widetilde{\varphi}^{2}(t)=\frac{1}{\sigma^{2}} e_{1}^{T} J_{N}^{-1} e_{1}
$$

and we arrive at (16) assuming all the DWO-optimal weights $w_{t}^{*}$ are positive. For the equidistant design (7), the results (18)-(19) now follow from straightforward calculations. 
Notice that for Gaussian $e(t)$ the DWO-optimal upper bound (16) coincides with the minimax lower bound (8) which means minimax optimality of the DWO-estimator among all estimators, not only among linear ones. For nonGaussian $e(t)$, similar optimality may be proved in a minimax sense over the class $\mathcal{Q}\left(\sigma^{2}\right)$ of all the regular densities $q(\cdot)$ of $e(t)$ with bounded variances

$$
E e^{2}(t) \leq \sigma^{2}
$$

As is well known, condition (26) implies

$$
I(q) \geq \sigma^{-2}
$$

Hence, see Remark 2.3, the lower bound

$$
\begin{gathered}
\sup _{q \in \mathcal{Q}\left(\sigma^{2}\right)} \sup _{f_{0} \in \mathcal{F}_{1}(M)} E_{f_{0}}\left(\tilde{f}_{N}-f_{0}\left(\varphi^{*}\right)\right)^{2} \\
\geq 4 M^{2}+e_{1}^{T} J_{N}^{-1} e_{1}
\end{gathered}
$$

follows directly from that of (8) with the same matrix $J_{N}$ as in (9).

From (21)-(25) we can derive a necessary and sufficient condition for the DWO-optimal weights to be positive, which can be explicitly written as

$$
\sum_{t=1}^{N} \varphi^{2}(t)-\varphi^{*} \sum_{t=1}^{N} \varphi(t)>\frac{1}{2}\left|\sum_{t=1}^{N} \varphi(t)-N \varphi^{*}\right|
$$

At least one point always satisfies (29), namely

$$
\varphi^{*}=\frac{1}{N} \sum_{t=1}^{N} \varphi(t)
$$

assuming that $J_{N}$ is non-degenerate. Thus, inequality (29) defines an interval of all those points $\varphi^{*}$ for which the DWO-optimal estimator is minimax optimal among all the estimators.

The (non-asymptotic) DWO-optimal weights $w_{t}^{*}$ will depend linearly on $\varphi(t)$, as directly seen from (21). Note also, that the analytic study of this subsection was possible to carry out since for the considered case the DWO-optimal weights are all positive, which led to a simpler, equivalent optimization problem (20), (5), having also a positive solution $w^{*}$. When there are also non-positive components in the solution of the problem (6), (5), an explicit analytic treatment is more difficult; it is considered below via approximating sums by integrals, for the equidistant design. In general, it can be shown that the weights satisfy

$$
w_{t}^{*}=\max \left\{\lambda_{1}+\mu \widetilde{\varphi}(t), 0\right\}+\min \left\{\lambda_{2}+\mu \widetilde{\varphi}(t), 0\right\}
$$

for some constants $\lambda_{1}<\lambda_{2}$ and $\mu$ (see (Roll et al., 2005b, Theorem 2) for a more general result).

\subsubsection{Both positive and non-positive weights}

In order to understand (at least on a qualitative level) what may happen when $w^{\text {opt }}$ contains both positive and negative components, let us assume 
equidistant design (7) and introduce the piecewise constant kernel functions $K_{w}:[-0.5,0.5] \rightarrow \mathbb{R}$ which correspond to an admissible vector $w:$

$$
\begin{aligned}
K_{w}(\varphi) & =\sum_{t=1}^{N} \mathbf{1}\{\varphi(t-1)<\varphi \leq \varphi(t)\} N w_{t}, \\
t & =1, \ldots, N
\end{aligned}
$$

where $\varphi(0)=-0.5$ and $\mathbf{1}\{\cdot\}$ stands for indicator. Now one may apply the following representations for the sums from (6), (5):

$$
\begin{aligned}
\sum_{t=1}^{N}\left|w_{t}\right| & =\int_{-0.5}^{0.5}\left|K_{w}(u)\right| d u \\
\sum_{t=1}^{N} w_{t}^{2} & =\frac{1}{N} \int_{-0.5}^{0.5} K_{w}^{2}(u) d u \\
\sum_{t=1}^{N} w_{t} & =\int_{-0.5}^{0.5} K_{w}(u) d u \\
\sum_{t=1}^{N} w_{t} \varphi(t) & =\int_{-0.5}^{0.5} u K_{w}(u) d u+O\left(N^{-1}\right)
\end{aligned}
$$

Thus, the initial optimization problem (6), (5) may asymptotically, as $N \rightarrow \infty$, be rewritten in the form of the following variational problem:

$$
\begin{aligned}
U_{N}(K)= & \frac{\sigma^{2}}{N} \int_{-0.5}^{0.5} K^{2}(u) d u \\
& +M^{2}\left(1+\int_{-0.5}^{0.5}|K(u)| d u\right)^{2} \rightarrow \min _{K}
\end{aligned}
$$

subject to constraints

$$
\int_{-0.5}^{0.5} K(u) d u=1, \quad \int_{-0.5}^{0.5} u K(u) d u=\varphi^{*} .
$$

Minimization in (37) is now meant to be over the admissible set $D_{0}$ that is the set of all piecewise continuous functions $K:[-0.5,0.5] \rightarrow \mathbb{R}$ meeting constraints (38). The solution to this problem is represented in the following assertion.

Assertion 2.3. Let $1 / 6<\varphi^{*}<1 / 2$. Then the asymptotically DWO-optimal kernel is

$$
K^{*}(u)=\frac{1}{h}\left(1+\frac{2}{h}(u-\Delta)\right) \mathbf{1}\{a \leq u \leq 0.5\}
$$

with

$$
h=\frac{3}{2}\left(1-2 \varphi^{*}\right), \quad \Delta=\frac{6 \varphi^{*}-1}{4}, \quad a=3 \varphi^{*}-1
$$

The DWO-optimal MSE upper bound becomes

$$
U_{N}\left(K^{*}\right)=4 M^{2}+\frac{\sigma^{2}}{N} \frac{8}{9\left(1-2 \varphi^{*}\right)},
$$


and the approximation to $w^{*}$ is given by

$$
w_{t}^{*} \approx \frac{1}{N} K^{*}\left(\varphi_{t}\right)
$$

Proof. See Nazin et al. (2003).

It is easily seen from (37) that asymptotically, as $N \rightarrow \infty$, the influence of the first summand in the RHS (37) becomes negligible, compared to the second one. Hence, we first need to minimize

$$
U_{N}^{(2)}(K)=\int_{-0.5}^{0.5}|K(u)| d u \rightarrow \min _{K \in D_{0}}
$$

However, the solution to (43) is not unique, and it is attained on any nonnegative kernel $K \in D_{0}$. A useful example of such a kernel is the uniform kernel function

$$
K_{u n i}^{*}(u)=\frac{1}{1-2 \varphi^{*}} \mathbf{1}\left\{\left|u-\varphi^{*}\right| \leq 1-\varphi^{*}\right\} .
$$

Here and below in the current subsection we assume that $0 \leq \varphi^{*}<1 / 2$, for the concreteness. It is straightforward to verify that $K_{u n i}^{*} \in D_{0}$, and

$$
U_{N}^{(1)}\left(K_{u n i}^{*}\right)=\int_{-0.5}^{0.5} K^{2}(u) d u=\frac{1}{1-2 \varphi^{*}}
$$

Let us compare this value $U_{N}^{(1)}\left(K_{u n i}^{*}\right)$ with that of $U_{N}^{(1)}\left(K^{*}\right)$ where the DWOoptimal kernel is known for $\left|\varphi^{*}\right| \leq 1 / 6$ to be

$$
K^{*}(u)=\left(1+12 \varphi^{*} u\right) \mathbf{1}\{|u| \leq 1 / 2\}
$$

The latter equation corresponds to (19) and may be obtained directly from (37)-(38) in a similar manner. Thus,

$$
U_{N}^{(1)}\left(K^{*}\right)=1+12 \varphi^{* 2}
$$

Figure 1 shows $U_{N}^{(1)}$ for the different kernels, as functions of $\varphi^{*}$.

Eq. (31) indicates that an optimal kernel $K^{*}$ might also contain a negative part. However, asymptotically (as $N \rightarrow \infty$ ), that may not occur since otherwise the main term of the MSE upper bound (37) - the second summand of the RHS (37) - is not minimized.

\section{Experiment Design}

Let us now briefly consider some experiment design issues. We first find and study the optimal design for a given estimation point $\varphi^{*} \in(-0.5,0.5)$ which minimizes the lower bound (8). Then a similar minimax solution is given for $\left|\varphi^{*}\right| \leq \delta$ with a given $\delta \in(0,0.5)$. 


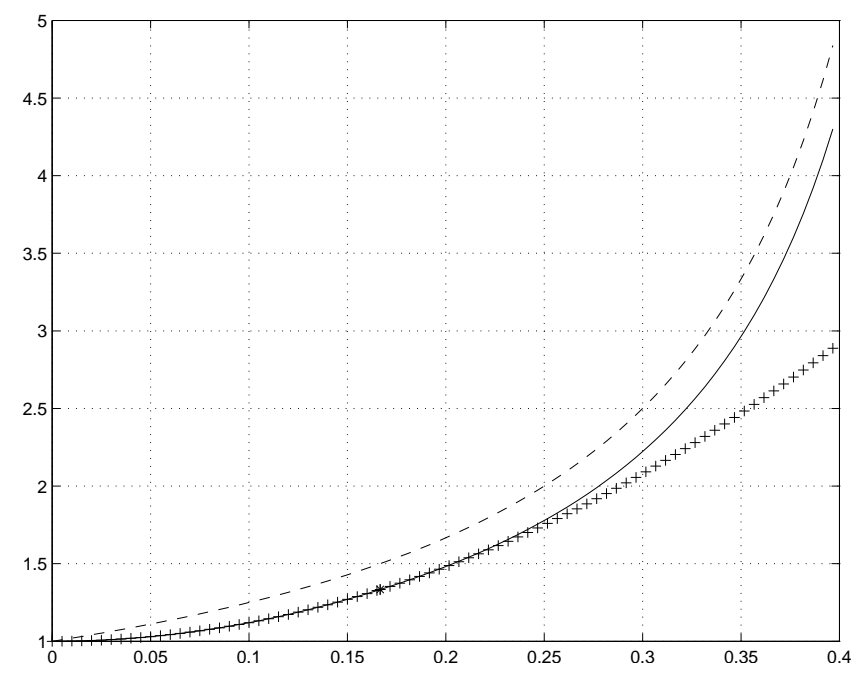

Figure 1: $U_{N}^{(1)}$ for DWO-optimal (solid) and uniform DWO-suboptimal (dashed) kernels; their minimax lower bound $1+12 \varphi^{* 2}$ is represented by plus signs; the point $\varphi^{*}=1 / 6$ is marked by a star.

\section{$3.1 \quad$ Fixed $\varphi^{*} \in(-0.5,0.5)$}

Let us fix $\varphi^{*} \in(-0.5,0.5)$ and minimize the lower bound (8) with respect to $\{\varphi(t)\}_{t=1}^{N}$ From (9), (22)-(25) follows that we are to minimize

$$
\lambda=\left(N-\frac{\left(\sum_{t=1}^{N} \widetilde{\varphi}(t)\right)^{2}}{\sum_{t=1}^{N} \widetilde{\varphi}^{2}(t)}\right)^{-1}
$$

which is equivalent to

$$
\begin{aligned}
& \frac{\left(S_{N}-N \varphi^{*}\right)^{2}}{V_{N}-2 \varphi^{*} S_{N}+N \varphi^{* 2}} \rightarrow \min _{|\varphi(t)| \leq 1 / 2} \\
& S_{N}=\sum_{t=1}^{N} \varphi(t), \quad V_{N}=\sum_{t=1}^{N} \varphi^{2}(t)
\end{aligned}
$$

Thus, the minimum in (49) equals zero and is attained on any design which meets the condition

$$
\frac{1}{N} S_{N}=\varphi^{*}
$$

One might find a design which maximizes $V_{N}$ subject to (50), arriving at the one of the form, for instance, $\varphi(t)= \pm 0.5$ with

$$
\#\{\varphi(t)=0.5\}=\frac{N}{2}\left(1+2 \varphi^{*}\right)
$$

and corresponding for $\#\{\varphi(t)=-0.5\}$, assuming the value in RHS (51) is an integer. Since $\lambda=1 / N$ and $\mu=0$ in (21), the DWO-optimal weights are 
uniform, $w_{t}^{*}=1 / N$. Hence, the upper and lower bounds coincide and equal

$$
U_{N}\left(w^{*}\right)=4 M^{2}+\frac{\sigma^{2}}{N}
$$

In general, however, the RHS of (51) is a non-integer. Then, one might take an integer part in (51), that is put $\#\{\varphi(t)=0.5\}=\left\lfloor 0.5 N\left(1+2 \varphi^{*}\right)\right\rfloor$ and $\#\{\varphi(t)=-0.5\}=N-\#\{\varphi(t)=0.5\}$, correcting also the value $\varphi(t)=0.5$ by a term $O(1 / N)$. Hence, we will have an additional term $O\left(N^{-2}\right)$ in the RHS (52).

\subsection{Minimax DWO-optimal Design}

Assume now $\left|\varphi^{*}\right| \leq \delta$ with $0<\delta \leq 0.5$, and, instead of (49), let us find a design solving

$$
\max _{\left|\varphi^{*}\right| \leq \delta} \frac{\left(S_{N}-N \varphi^{*}\right)^{2}}{V_{N}-2 \varphi^{*} S_{N}+N \varphi^{* 2}} \rightarrow \min _{|\varphi(t)| \leq 1 / 2}
$$

The maximum in (53) can be explicitly calculated, giving

$$
\frac{\left(\left|S_{N}\right|+N \delta\right)^{2}}{V_{N}+2 \delta\left|S_{N}\right|+N \delta^{2}} \rightarrow \min _{|\varphi(t)| \leq 1 / 2}
$$

Evidently, the LHS of (54) is monotone decreasing w.r.t. $V_{N}$ and monotone increasing w.r.t. $\left|S_{N}\right|$. Hence, the minimum in (53) would be attained if $V_{N}=$ $N / 4$ (that is its upper bound) and if $S_{N}=0$. Assuming that $N$ is even, these extremal values for $V_{N}$ and $\left|S_{N}\right|$ are attained under the symmetric design $\varphi(t)= \pm 0.5$ with

$$
\#\{\varphi(t)=0.5\}=\#\{\varphi(t)=-0.5\}=\frac{N}{2}
$$

This design ensures the minimax of the DWO-optimal MSE

$$
\min _{|\varphi(t)| \leq 1 / 2} \max _{\left|\varphi^{*}\right| \leq \delta} U_{N}\left(w^{*}\right)=4 M^{2}+\frac{\sigma^{2}}{N}\left(1+4 \delta^{2}\right)
$$

Particularly, for $\delta=1 / 2$,

$$
\min _{|\varphi(t)| \leq 1 / 2} \max _{\left|\varphi^{*}\right| \leq 1 / 2} U_{N}\left(w^{*}\right)=4 M^{2}+\frac{2 \sigma^{2}}{N}
$$

Putting $\delta=0$ in (56) yields (52) with $\varphi^{*}=0$.

Now, if we apply this design for an arbitrary $\varphi^{*} \in(-0.5,0.5)$, we arrive at the DWO-optimal MSE

$$
U_{N}\left(w^{*}\right)=4 M^{2}+\frac{\sigma^{2}}{N}\left(1+4 \varphi^{* 2}\right)
$$

with the DWO-optimal weights

$$
w_{t}^{*}=\frac{1}{N}\left(1+4 \varphi^{*} \varphi(t)\right)
$$

which are all positive. Hence, the upper bound (58) coincides with the lower bound (8), and the DWO estimator with weights (59) is minimax optimal for any $\varphi^{*} \in(-0.5,0.5)$. For the odd sample size $N$, one may slightly correct the design, arriving at an additional term $O\left(N^{-2}\right)$ in the RHS (58), similarly to the previous subsection. 


\section{Conclusions}

In this paper, the DWO approach has been studied for the class of approximately linear functions, as defined by (2). A lower bound on the maximum MSE for any estimator was given, and it was shown that this bound is attained by the DWO estimator if the DWO-optimal weights are all positive. This means that the DWO estimator is optimal among all estimators for these cases. As we can see from (29)-(30), there is always at least one $\varphi^{*}$ (and hence an interval) for which this is the case, as long as the information matrix is non-degenerate. For the optimal experiment designs considered in Section 3, the corresponding DWO estimators are always minimax optimal.

\section{References}

A. V. Gol'denshlyuger and A. V. Nazin. Parameter estimation under random and bounded noises. Automation and Remote Control, 53(10, pt. 1):1536$1542,1992$.

C. Harris, X. Hong, and Q. Gan. Adaptive Modelling, Estimation and Fusion from Data: A Neurofuzzy Approach. Springer-Verlag, 2002.

A. Juditsky, H. Hjalmarsson, A. Benveniste, B. Delyon, L. Ljung, J. Sjöberg, and Q. Zhang. Nonlinear black-box modeling in system identification: Mathematical foundations. Automatica, 31(12):1724-1750, 1995.

V. Ya. Katkovnik and A. V. Nazin. Minimax lower bound for time-varying frequency estimation of harmonic signal. IEEE Trans. Signal Processing, 46 (12):3235-3245, December 1998.

A. Nazin, J. Roll, and L. Ljung. A study of the DWO approach to function estimation at a given point: Approximately constant and approximately linear function classes. Technical Report LiTH-ISY-R-2578, Dept. of EE, Linköping Univ., Sweden, December 2003.

A. S. Nemirovskii. Recursive estimation of parameters of linear plants. Automation and Remote Control, 42(4, pt. 6):775-783, 1981.

J. Roll. Local and Piecewise Affine Approaches to System Identification. PhD thesis, Dept. of EE, Linköping Univ., Sweden, April 2003.

J. Roll, A. Nazin, and L. Ljung. A non-asymptotic approach to local modelling. In The 41st IEEE Conference on Decision and Control, pages 638-643, December 2002.

J. Roll, A. Nazin, and L. Ljung. Nonlinear system identification via direct weight optimization. Automatica: Special Issue on Data-Based Modelling and System Identification, 41(3):475-490, March 2005a.

J. Roll, A. Nazin, and L. Ljung. A general direct weight optimization framework for nonlinear system identification. In 16th IFAC World Congress on Automatic Control, Prague, Czech Republic, July 2005b. 
J. Sacks and D. Ylvisaker. Linear estimation for approximately linear models. The Annals of Statistics, 6(5):1122-1137, 1978.

J. Sjöberg, Q. Zhang, L. Ljung, A. Benveniste, B. Delyon, P. Y. Glorennec, H. Hjalmarsson, and A. Juditsky. Nonlinear black-box modeling in system identification: a unified overview. Automatica, 31(12):1691-1724, 1995.

J. A. K. Suykens, T. van Gestel, J. De Brabanter, B. De Moor, and J. Vandewalle. Least Squares Support Vector Machines. World Scientific, Singapore, 2002 .

\section{A Auxiliary Information Lower Bounds}

The following lemma as well as its proof goes back to the arguments by Nemirovskii Nemirovskii (1981) which were further adopted in Gol'denshlyuger and Nazin (1992) to a particular problem of parameter estimation under both random and non-random but bounded noise; see also Katkovnik and Nazin (1998) and the references therein. The proofs for both lemmas in this section can be found in Nazin et al. (2003).

Lemma A.1. Let $\widetilde{\theta}_{N}: \mathbb{R}^{N} \rightarrow \mathbb{R}^{2}$ be an arbitrary estimator for $\theta \in \mathbb{R}^{2}$, based on a dataset $\{\varphi(k), y(k)\}_{k=1}^{N}$ with observations

$$
y(k)=\theta^{T} F(k)+r+e(k), \quad k=1, \ldots, N
$$

with fixed regressors $F(k)=\left(\begin{array}{ll}1 & \varphi(k)\end{array}\right)^{T}, \varphi(k) \in \mathbb{R}$, the noise e $(k)$ being i.i.d. Gaussian $N\left(0, \sigma^{2}\right)$, and $|r| \leq \varepsilon$. Then for any $h=\left(\begin{array}{ll}h_{1} & h_{2}\end{array}\right)^{T} \in \mathbb{R}^{2}$, the following information inequality holds

$$
\sup _{\theta} \sup _{|r| \leq \varepsilon} E_{\theta, r}\left(h^{T}\left(\tilde{\theta}_{N}-\theta\right)\right)^{2} \geq\left(\varepsilon h_{1}\right)^{2}+h^{T} J_{N}^{-1} h
$$

with the Fisher information matrix

$$
J_{N}=\frac{1}{\sigma^{2}} \sum_{k=1}^{N} F(k) F^{T}(k)
$$

which is supposed to be invertible.

Lemma A.2. Let $\widetilde{\theta}_{N}: \mathbb{R}^{N} \rightarrow \mathbb{R}^{2}$ be an arbitrary estimator for $\theta \in \mathbb{R}^{2}$, based on observations (60), but with

1) regressors $F(k)=\left(\begin{array}{ll}1 & \varphi(k)-\varphi^{*}\end{array}\right)^{T}$ having random i.i.d. entries $\varphi(k)$ uniformly distributed on the interval $[-0.5,0.5]$;

2) i.i.d. Gaussian random noise $e(k) \in N\left(0, \sigma^{2}\right)$;

3) $\{e(k)\}_{k=1}^{N}$ and $\{\varphi(k)\}_{k=1}^{N}$ independent;

4) finally, $|r| \leq \varepsilon$.

Then, for any $h=\left(h_{1}, \quad h_{2}\right)^{T} \in \mathbb{R}^{2}$, (61) holds with the Fisher information matrix

$$
J_{N}=\frac{N}{\sigma^{2}}\left(\begin{array}{cc}
1 & -\varphi^{*} \\
-\varphi^{*} & \varphi^{* 2} / 12
\end{array}\right)
$$




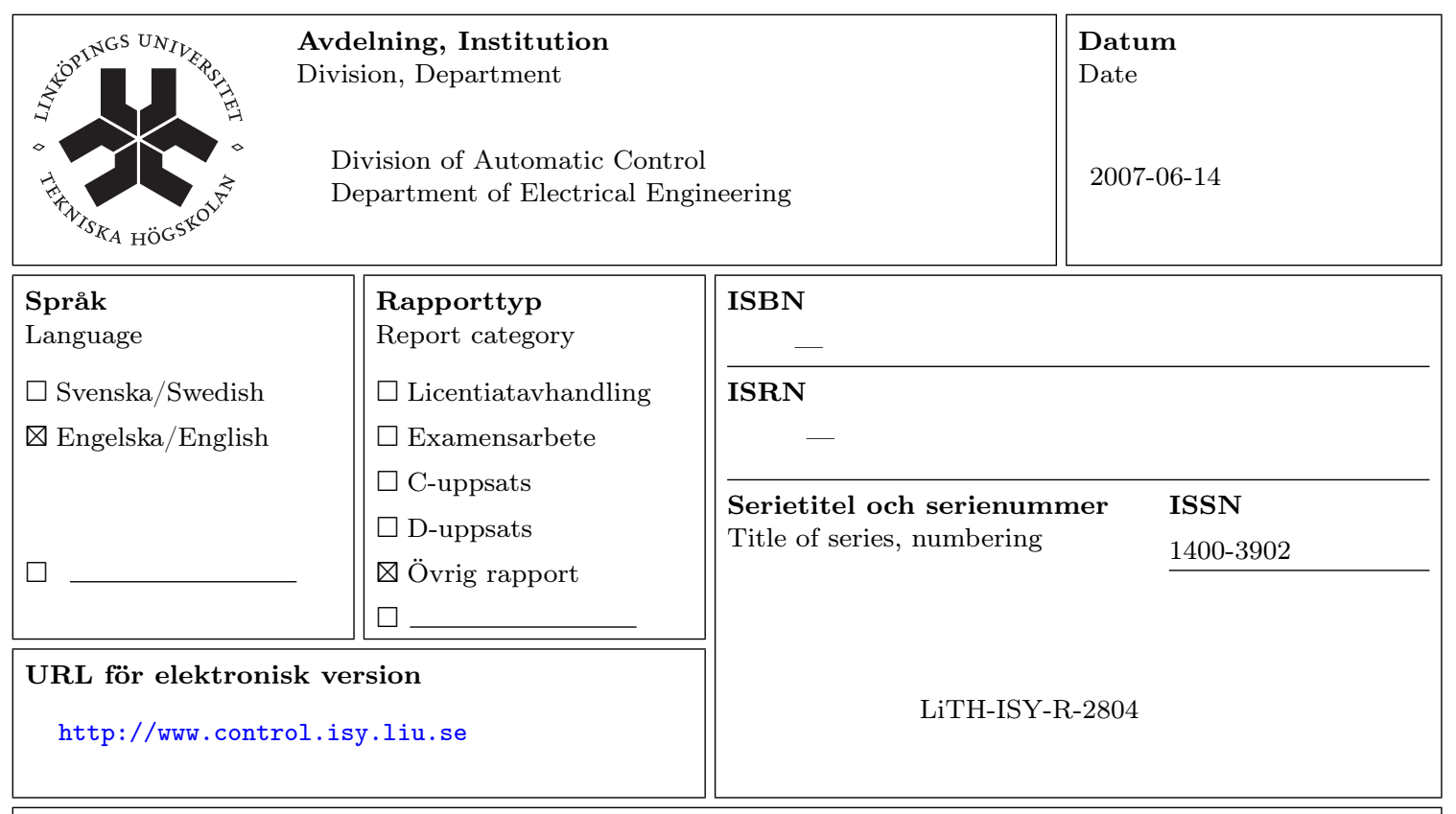

Titel Direct Weight Optimization for Approximately Linear Functions: Optimality and Design Title

Författare Alexander Nazin, Jacob Roll, Lennart Ljung

Author

\section{Sammanfattning}

Abstract

The Direct Weight Optimization (DWO) approach to estimating a regression function is studied here for the class of approximately linear functions, i.e., functions whose deviation from an affine function is bounded by a known constant. Upper and lower bounds for the asymptotic maximum MSE are given, some of which also hold in the non-asymptotic case and for an arbitrary fixed design. Their coincidence is then studied. Particularly, under mild conditions, it can be shown that there is always an interval in which the DWO-optimal estimator is optimal among all estimators. Experiment design issues are also studied. 